\title{
Cervical Cancer - A Preventable and Potentially Curable Cancer
}

Cancer is predicted to be an increasingly important cause of morbidity and mortality in Bangladesh in the next few decades. The estimated incidence of 12.7 million new cancer cases will rise to 21.4 million by $2030^{1}$.Cervical cancer is the fourth most frequent cancer in women with an estimated 570,000 new cases in 2018 representing $7.5 \%$ of all female cancers and one women dying every 2 minutes $^{2}$. Approximately, $90 \%$ of deaths from cervical cancer occurred in low and middle-income countries. It is the 2nd most common cancer among women in Bangladesh, and every year 11,956 new cases are detected and 6582 women $\mathrm{die}^{3}$. Human Papilloma Virus (HPV) has now been identified as the etiological agent responsible for cervical cancer. HPV prevalence increases with high risk sexual behaviour and poor sexual hygiene ${ }^{4}$. About $90 \%$ of HPV clear within 2 years and a small proportion of infections can persist and progress to cervical cancer in 15 to 20 years $^{5}$.

But the truth is that almost all of these deaths could be avoided if all adolescent girls were immunized against HPV, cervical screening and treatment of pre-cancerous lesions were available to all women. There are currently vaccines available protecting against $90 \%$ of cervical cancers. A HPV vaccination trial was conducted in Bangabandhu Sheik Mujib Medical University in $2008^{6}$. and Govt. initiated HPV vaccination in 2016 and scaling up the programme to girls of grade $\mathrm{V}$ at school and 10 years at community ${ }^{3}$.

Early diagnosis and effective treatment can significantly improve the likelihood of survival. WHO Package of essential non-communicable (PEN) disease interventions for primary health care in low-resource settings included referral of women with suspected cervical cancer ${ }^{7}$. A successful initiative was taken in Korail Slum, Dhaka, Bangladesh by Massachusetts General Hospital, Boston, USA and AK Khan Health Care Trust, Dhaka, Bangladesh in 2011 with both screenand-treat precancerous lesions with visual inspection of cervix with acetic acid (VIA) and loop electrosurgical excision procedure (LEEP) ${ }^{8}$. According to National Cervical Cancer Strategy, Bangladesh gradually developed opportunistic screening facilities with VIA for the women above 30 years. All screen-positive women will be counseled, further evaluated by colposcopy and treated at appropriate facilities ${ }^{3}$. Three types of screening tests currently recommended by WHO : HPV testing for high-risk HPV types, VIA, Pap test and liquid-based cytology (LBC), for treatment of pre-cancer lesions, WHO recommends treatment for precancerous lesion of cryotherapy and LEEP. For advanced lesions, women should be referred for further investigations and adequate management ${ }^{5}$.

There are five types of standard treatment: Surgery, Radiation therapy, Chemotherapy, Targeted therapy and Immunotherapy. Radiation therapy uses high-energy xrays to kill cancer cells. External radiation therapy includes 3-dimensional radiation therapy, Intensity-modulated radiation therapy and Volumetric Arc Therapy, Stereotactic Radiosurgery. Internal radiation therapy (brachytherapy) uses a radioactive substance sealed in needles, seeds, wires, or catheters that are placed directly into or near the cancer. Chemotherapy is a cancer treatment that uses drugs to stop the growth of cancer cells, either by killing the cells or by stopping them from dividing. The way the chemotherapy is given depends on the type and stage of the cancer being treated.

Cancer research is vital to find new management strategies. Some research works are also going on in Bangladesh. Recently, a promising research work has performed in National Cancer Research Institute and Hospital, Dhaka, Bangladesh on prognostic impact of histopathological type of cervical cancer on disease free survival (DFS), local, loco-regional and distal recurrence after completion of treatment with radiation therapy. In multivariate analysis it was shown that histopathological type and stage of disease were found to be independently significant prognostic factors for DFS.

It is expected that prevention and cure of cervical cancer will be possible in near future.

(J Bangladesh Coll Phys Surg 2019; 37: 167-168) DOI: http://dx.doi.org/10.3329/jbcps.v37i.43344

\section{Prof Syed Akram Hussain}

Coordinator, Square Oncology and Radiotherapy Centre Dhaka, Bangladesh 


\section{References:}

1. Syed Akram Hussain and Richard Sullivan, Cancer Control in Bangladesh, Jpn J Clin Oncol. 2013 Dec; 43(12): 1159 1169.

2. Cervical Cancer. https://www.who.int/cancer/prevention/ diagnosis-screening/cervical-cancer/en.

3. National Strategy for Cervical Cancer prevention and control Bangladesh (2017-2022). (https : // www.searo.who.int > entity > cervical-cancer-strategyenglish-book250118).

4. National Cancer Control Strategy and Plan of Action 200915. Directorate General of Health Services, Ministry of Health and Family Welfare, Bangladesh (http: // www.ban.searo.who.int/linkfiles/publication cancer Startegy.pdf.pdf.)

5. Human Papilloma Virus and Cervical Cancer. https:// www.who.int > Newsroom > Fact sheets > Detail.
6. Sabera Khatun, Syed Md Akram Hussain, Sameena Chowdhury,Jannatul Ferdous,Fawzia Hossain,Sultana Razia Begum. Safety and Immunogenicity Profile of Human Papillomavirus-16/18 AS04 Adjuvant Cervical Cancer Vaccine: A Randomized Controlled Trial in Healthy Adolescent Girls of Bangladesh, Jpn J Clin Oncol. 2012 Jan; 42(1): 36-41.

7. Package of Essential Noncommunicable (PEN) Disease Interventions for Primary Health Care in Low-Resource Setting. (https://www.who.int/nmh/publications/essential_ ncd_interventions_lr_settings.pdf).

8. Leslie S. Bradford, Bimalangshu R. Dey, Syed Md Akram Hussain, Sultana Razia Begum, Farhat Hussain, Sharmina Hoque, Annekathryn Goodman. Development of a cervical cancer screening program in a slum setting using visual inspection with acetic acid: Analysis of feasibility and cost. Open Journal of Obstetrics and Gynecology, 2012, 2, 140-146. 